\title{
PROPIEDAD INTELECTUAL EN EL DEPORTE: TENDENCIAS EN PATENTES DE LA INDUSTRIA DEPORTIVA INTERNACIONAL Y COLOMBIANA
}

\author{
PROPRIEDADE INTELECTUAL NO DESPORTO: \\ TENDÊNCIAS EM PATENTES DA INDÚSTRIA \\ ESPORTIVA INTERNACIONAL E COLOMBIANA
}

\section{INTELLECTUAL PROPERTY IN SPORTS: TRENDS IN PATENTS OF THE INTERNATIONAL AND COLOMBIAN SPORTS INDUSTRY}

\author{
Dario José Espinal Ruiz ${ }^{1}$ \\ Javier Enrique Medina Vásquez ${ }^{2}$ \\ Flávia da Cunha Bastos ${ }^{3}$
}

1 Estudiante de doctorado Colciencias-Universidad del Valle. Facultad de ciencias de la administraci $\mathrm{n}$. Grupo de investigaci $\mathrm{n}$ previsi $\mathrm{n}$ y pensamiento estrat gico. L nea de investigaci $n$ en prospectiva y estrategia. Profesional en cultura $f$ sica y deportes-universidad INCCA de Colombia. Candidato a doctor en administraci n universidad del valle. E-mail: dario.espinal@correo univalle.edu.co

2 Vicerrector de investigaciones-Profesor titular -Universidad del Valle. Facultad de ciencias de la administraci $n$. Grupo de investigaci $n$ previsi $n$ y pensamiento estrat gico. $L$ nea de investigaci $n$ en prospectiva y estrategia. Psic logo-Universidad del Valle. Doctor en ciencias sociales-Universidad gregoriana de Roma. E-mail: javier.medina@correounivalle.edu.co 
3 Profesora Asistente Universidad de S o Paulo. Departamento de Deporte de la escuela de educaci $\mathrm{n}$ f sica y deporte - Universidad de $\mathrm{S}$ o Paulo. Grupo de estudios e investigaci $\mathrm{n}$ en gesti $\mathrm{n}$ del deporte-Universidad del $\mathrm{S}$ o Paulo. Licenciada en Educaci n F sica- Universidad del S o Paulo. Doctora en educaci n-Universidad de S o Paulo. E-mail: flaviacb@usp.br

Espinal Ruiz, D. J.; Medina V squez, J. E. \& da Cunha Bastos, F. (2017). Propiedad Intelectual en el Deporte: Tendencias en Patentes de la Industria Deportiva Internacional y Colombiana. Educaci n F sica y Deporte, 36(1), 21-40, Ene.-Jun. http://doi. org/10.17533/udea.efyd.v36n1a02 


\section{RESUMEN}

La propiedad intelectual en el deporte es un tema poco estudiado, por lo que se carece de informaci $\mathrm{n}$ robusta para describir el panorama global de la innovaci $\mathrm{n}$ de la industria deportiva. El presente art culo tiene como prop sito identificar las tendencias de la industria deportiva internacional y la realidad colombiana sobre la protecci $n$ de la innovaci $n$ en el mercado de patentes. Mediante un estudio exploratorio, se caracterizaron los indicadores bibliom tricos para patentes en los Itimos 20 a os. Los resultados muestran un aumento notable desde 2007 en registros de patentes, siendo China el pas m s innovador, y el mercado de aparatos para el entrenamiento $f$ sico el de mayor crecimiento a nivel global. En Colombia se registran 17 familias de patentes y seis segmentos estrat gicos para el desarrollo de productos altamente innovadores.

PALABRAS CLAVE: Innovaci n deportiva, desarrollo deportivo, organizaciones deportivas, prospectiva y vigilancia tecnol gica.

\section{RESUMO}

A propriedade intelectual no desporto um tema pouco estudado no desporto, portanto, carece-se de informaç o robusta para descrever o panorama global da inovaç o da ind stria esportiva. O presente artigo tem como prop sito identificar as tend ncias da ind stria esportiva internacional e a realidade colombiana sobre a proteç o da inovaç o no mercado de patentes. Mediante um estudo explorat rio caracterizaram-se os indicadores bibliom tricos para patentes nos Itimos 20 anos. Os resultados mostram um aumento not vel desde o ano 2007 em registros de patentes sendo China o pas mais inovador, o mercado dos aparelhos para o treinamento $\mathrm{f}$ sico o de maior crescimento a n vel global. Em Col mbia, registram-se 17 fam lias de patentes e seis segmentos estrat gicos para o desenvolvimento de produtos altamente inovadores.

PALAVRAS-CHAVE: Inovaç o esportiva, desenvolvimento esportivo, organizaç es esportivas, prospectiva e vigil ncia tecnol gica.

\section{ABSTRACT}

Intellectual property in sports is a little studied subject, therefore, there is a lack of relevant information to describe the global panorama of innovation in the sports industry. This article aims to identify the trends in the international sports industry and the Colombian reality about the protection of innovation in the patent market. Through an exploratory study, bibliometric indicators were characterized for patents in the last 20 years. The results show a remarkable increase since 2007 in patent registrations being China 
the most innovative country; the market of the devices for physical training devices presents the greater growth at a global level. In Colombia, 17 patent families and six strategic segments are registered for the development of highly innovative products.

KEY WORDS: Sports innovation, sports development, sports organizations, foresight and technological surveillance. 


\section{INTRODUCCIÓN}

El deporte es, por naturaleza, innovador, debido a su capacidad de adaptarse $r$ pidamente a las nuevas tendencias sociales y tecnol gicas(Ratten \& Ferreira, 2016) (Ratten \& Ferreira, 2016) (Ratten \& Ferreira, 2016). En este contexto, se realizan innovaciones en dos reas: nuevos productos deportivos (desarrollo de nuevos materiales, implementos, indumentaria, nuevas tecnolog as), y nuevos servicios deportivos (incremento de la satisfacci $\mathrm{n}$ de los usuarios actuales, reglamentarias y programas para aumentar la base de participaci $n$ y vinculaci $n$ al deporte) (Winand \& Hoeber, 2016; Tjønndal, 2016).

Las estad sticas de patentes, como indicadores de resultado (output), son la fuente de informaci $\mathrm{n} \mathrm{m}$ s completa y accesible para el an lisis de los niveles de innovaci $n$ de una industria (de Beer, 2015). Sin embargo, ha sido un tema poco estudiado en el contexto del deporte ( Ratten, 2011; Schaefer, 2012; Ratten \& Ferreira, 2016); por consiguiente, se carece de informaci $n$ robusta para describir el panorama de la protecci $n$ de la innovaci $\mathrm{n}$ en la industria deportiva internacional y colombiana. Frente a este escenario, el presente art culo tiene como prop sito identificar las tendencias en el mercado de patentes de la industria deportiva internacional y colombiana en los Itimos 20 a os (1997-2017), y determinar los segmentos estrat gicos con mayor potencial para el desarrollo de productos deportivos altamente innovadores de origen colombiano.

\section{Propiedad intelectual en el deporte}

El alto valor comercial que poseen las organizaciones deportivas, ha exigido la prevenci $\mathrm{n}$ de la explotaci $\mathrm{n}$ ilegal por parte de terceros. La propiedad intelectual es el ordenamiento legal que tutela las producciones cient ficas, literarias, art sticas y desarrollos tecnol gicos, como resultado del talento humano, siempre que sean susceptibles de plasmarse en cualquier tipo 
de soporte, medio de producci $n$, reproducci $n$ o divulgaci $n$ conocido o por conocer (Ceballos, 2014).

La organizaci $n$ mundial de propiedad intelectual (WIPO) distingue dos ramas de la propiedad intelectual: la propiedad industrial y los derechos de autor. La propiedad industrial se refiere a patentes de invenci $n$, modelos de utilidad, dise os industriales, secretos comerciales, esquemas de trazados de circuitos integrados, y signos distintivos, como marcas, marcas de origen, lemas comerciales, nombres comerciales, $r$ tulos, ense-

as, o por cualquier otra forma que llegue a regularse en esta materia por normas posteriores. Por otro lado, se establece que son objeto de derechos de autor (copyright), toda producci $n$ del dominio cient fico, literario y art stico que pueda reproducirse o definirse por cualquier forma de impresi $\mathrm{n}$ o de reproducci $n$, por fonograf a, radiotelefon a o cualquier otro medio conocido, o por conocer (CAN, 1993; Universidad del Valle, 2003; WIPO, 2016).

La propiedad intelectual en el deporte tiene una amplia aplicaci n. Las patentes fomentan los avances tecnol gicos que, a su vez, traen como resultado mejores materiales, implementos, accesorios e indumentarias; el patrocinio, las marcas registradas y los dise os, confieren una identidad distintiva a los eventos, equipos, deportistas y consumidores; los derechos conexos al derecho de autor permiten generar los ingresos necesarios para que los medios de comunicaci $n$ inviertan en la difusi $n$ de eventos deportivos; los derechos de propiedad intelectual son objeto de acuerdos de comercializaci $n$ y concesi $n$ de licencias que, a su vez, generan ingresos que fomentan la industria del deporte, los secretos profesionales, los derechos sobre la imagen de deportistas (Comerford, 2012; Kalamadi, 2012; Schaefer, 2012).

La patente es el derecho otorgado a un inventor por un Estado, o por una oficina regional que act a en nombre de varios Estados, que permite al inventor impedir a terceros ex- 
plotar por medios comerciales su invenci $\mathrm{n}$ durante un plazo limitado, que suele ser de 20 a os. Las patentes deben cumplir las siguientes condiciones de patentabilidad: la invenci $\mathrm{n}$ debe tener utilidad pr ctica y/o ser susceptible aplicaci n industrial; debe observarse una invenci n no conocida en el estado de la t cnica; la actividad inventiva no puede ser deducida por una persona con conocimientos generales en el campo espec fico de la t cnica; y debe ser de materia patentable, conforme a la normatividad de cada pa s (WIPO, 2016).

El ndice global de innovaci $n$, el ndice global de propiedad intelectual y el ndice global de competitividad, son algunos de los sistemas de clasificaci $\mathrm{n}$ internacional $\mathrm{m}$ s relevantes para la formulaci $\mathrm{n}$ de pol ticas de propiedad intelectual. Estos ndices utilizan las estad sticas de patentes como indicadores de resultados (output) de la innovaci n como uno de los factores que contribuyen a la competitividad de las naciones (Dutta et al., 2016; Taylor, 2016; Schwab \& Sala-i-Martin, 2016).

Las patentes en el campo del deporte se han concentrado en tres categor as: el juzgamiento de competencias deportivas, el rendimiento y desempe o de atletas, y los aspectos relacionados con la seguridad (Ming \& Si Hu, 2013). Las patentes de proceso han adquirido una gran relevancia en el deporte; existe una gran cantidad de $\mathrm{m}$ todos de entrenamiento que han sido protegidos con patentes en Estados Unidos, como putting a golf ball ${ }^{1}$, fitness training method $s^{2}$, training baseball pitchers ${ }^{3}$, training swings ${ }^{4}$, entre otros (Kief et al., 2009).

Kief et al. (2009) sugieren al menos tres formas de patente de proceso que se han utilizado para asegurar los derechos sobre los movimientos deportivos. En primer lugar, la protec-

\footnotetext{
1 US Patent 5,616,089 (29 de marzo de 1996).

2 US Patent 6,190,291 (20 de febrero de 2001).

3 US Patent5,639,243 (17 de junio de 1997).

4 US Patent 6,176, 790 (23 de junio de 2001).
} 
ci $\mathrm{n}$ de patentes sobre movimientos que obtienen resultados exitosos, tales como carreras $\mathrm{m}$ s $\mathrm{r}$ pidas o saltos $\mathrm{m}$ s largos. En segundo lugar, la protecci $\mathrm{n}$ de derechos de autor para movimientos que son creativos, como las coreograf as gimn sticas o de patinaje art stico sobre hielo. Finalmente, el registro de marcas que indican que los movimientos provienen de una fuente nica de bienes o servicios, como el air Jordan slam dunk.

\section{Tendencias actuales y futuras de la industria deportiva internacional}

La industria del deporte est constituida por m s de 400 actividades econ micas (MCVET, 2008; EC, 2013). Su definici $n$ debe partir de lo que en cada territorio se considera como deporte, ya que, de acuerdo a esto, se asumen los diferentes bienes y servicios que la integran (Milano \& Chelladurai, 2011). Espinal (2018) define la industria del deporte como una red productiva que tiene como prop sito el desarrollo tecnol gico y la innovaci $n$ en bienes, servicios y eventos deportivos, se encuentra establecida en un territorio, y est formada por una variedad de empresas, organizaciones e instituciones que se benefician de una pol tica p blica y un marco jur dico para promover su crecimiento, de un mercado laboral especializado y un conjunto de infraestructuras $\mathrm{p}$ blicas y privadas que la soportan.

En la literatura se identifican, para la industria del deporte, tres tipos de aglomeraciones o cl steres: los relativos a los servicios, a la producci $\mathrm{n}$ de bienes y aquellos que se basan en la organizaci n y promoci n de eventos (Zheng, 2011). Estos cl steres se encuentran concentrados en 54 ciudades de 14 pa ses (2 de Norteam rica, 1 de Ocean a, 4 de Europa, 7 de Asia, y ninguno de Latinoam rica y frica) (Estrada, 2015).

El crecimiento y desarrollo de una industria, es directamente proporcional a la capacidad de protecci $\mathrm{n}$ de sus innovaciones (Mateska, 2016; de Beer, 2016); esto se manifiesta en los ingresos mundiales generados por la propiedad intelectual en 
la industria del deporte internacional. Los acuerdos de patrocinio, los beneficios de taquilla, los derechos de comunicaci $n$ y el merchandising, ascendieron a los 145.000 millones USD en 2015 (PWC, 2015). Alrededor del mundo, la industria del deporte obtiene ingresos entre 480.000 y 620.000 millones USD, incluyendo implementos, indumentaria y calzado (Herstein \& Berger, 2013). Otras estimaciones, afirman que el valor de la industria del deporte a nivel global es de un trill $\mathrm{n}$ doscientos mil millones de d lares (Plunkett, 2017).

Las tendencias internacionales, en $\mathrm{t}$ rminos de segmentos de mercado, tienen como el de mayor participaci $\mathrm{n}$ a los deportes de aventura $(23,3 \%)$, seguido de los deportes de pelota $(22,9 \%)$ y del fitness $(18,3 \%)$. Por regiones, la distribuci $n$ del mercado de bienes deportivos se concentra en Estados Unidos $(31,9 \%)$, Europa $(30,2 \%)$ y Asia $(28,1)$. Durante el periodo 2009 2014, el crecimiento del mercado de bienes deportivos se concentr principalmente en Asia (4,3\%), Estados Unidos (1,8\%) y Europa $(1,4 \%)$. El segmento de mayor crecimiento en Asia fue el del Golf $(27,6 \%)$, en Estados Unidos fueron los deportes de aventura $(37,8 \%)$, y en Europa fueron los deportes de pelota $(24,8 \%)$. Se estima que el mercado deportivo crecer al 2,9\% anual hasta el a o 2018 (Marketline, 2014).

Las tendencias futuras de la industria del deporte se orientan hacia los avances en las tecnolog as de la comunicaci $n$ y los transportes sostenibles; en la eliminaci $\mathrm{n}$ de las barreras geogr ficas, culturales y de intercambio comercial de la oferta deportiva; en la diversificaci $n$ de los servicios deportivos y su forma de acceso a trav $\mathrm{s}$ de la realidad virtual y la realidad aumentada; en la gesti $n$ y monitoreo del entrenamiento y la competencia deportiva basado en la inteligencia artificial; en la mejora del desempe o deportivo por medio de la ingenier a gen tica y la biotecnolog a; $y$ en la introducci $n$ de competencias virtuales, rob ticas y de ciborgs, entre otros (Li et al., 2012; Harari, 2016). 


\section{METODOLOGÍA}

La aplicaci $\mathrm{n}$ de indicadores bibliom tricos a las bases de datos de patentes (patentometr a) es la t cnica m s efectiva para comprobar el estado actual de la tecnolog a, identificar tendencias emergentes y descubrir nichos de mercado (D az et al., 2007). Para lograr los objetivos propuestos, se realiz un estudio de tipo exploratorio (Skinner et al., 2015), siguiendo la metodolog a de la vigilancia tecnol gica basada en la t cnica del an lisis de patentes (Cantrell, 1999).

Se identificaron las tendencias en la innovaci $\mathrm{n}$ tecnol gica de la industria deportiva internacional y colombiana en la metabase de datos de patentes denominada Patbase Analytics, que contiene la informaci $\mathrm{n}$ de todas las patentes registradas a nivel global, en cada una de las agencias de m s de 100 pa ses y oficinas de patentes $\mathrm{m} \mathrm{s}$ importantes del mundo.

El universo de $b$ squeda fueron todos los registros de patentes relacionados con el deporte a nivel mundial y en Colombia, en los Itimos 20 a os (1997-2017). Se utiliz la siguiente ecuaci $n$ de b squeda como $\mathrm{c}$ digo para filtrar los resultados:

$((T A=($ SPORT $\sim$ OR SPORTS NOT TRANSPORT $)))$ AND (PD>1996)

Esta ecuaci $\mathrm{n}$ fue validada por un grupo de 5 expertos, y se prob en un primer periodo de vigilancia tecnol gica de 9 meses previos a los resultados finales expuestos en este art culo (ver tabla 1).

El procesamiento, an lisis y visualizaci $n$ de la informaci $n$, fue interpretado seg $\mathrm{n}$ los siguientes indicadores descriptivos de tipo bibliom trico para el an lisis de patentes: $n$ mero total de familias de patentes, $\mathrm{n}$ mero total de familias de patentes por jurisdicci $n, n$ mero total de familias de patentes por a o, tipos de familias de patentes, y $\mathrm{n}$ mero total de familias de patentes por cesionarios. 
Tabla 1. Bitácora de búsqueda

\begin{tabular}{ll}
\hline Bitácora & Datos de referencia \\
\hline Validación (vigilancia) & 17 de febrero de 2017 \\
Fecha de búsqueda final & 8 de noviembre de 2017 \\
Software de búsqueda & Patbase Analytics \\
Análisis de búsqueda & Los autores \\
Campo de búsqueda & Patentes \\
Ecuación de búsqueda & $(($ TA $=($ SPORT OR SPORTS NOT \\
Periodo de tiempo de la búsqueda & TRANSPORT)) AND (PD>1996) \\
Alcance de la búsqueda & Mundial-Colombia \\
\hline
\end{tabular}

Fuente: elaboración propia

Los I mites de este estudio se establecen exclusivamente sobre la informaci $\mathrm{n}$ en el registro de patentes de la base de datos global Patbase Analytics, disponibles durante la fecha establecida en la bit cora de b squeda planeada. Este estudio no represento ning $\mathrm{n}$ riesgo de tipo tico durante el proceso investigativo.

\section{RESULTADOS}

De acuerdo con la ecuaci $\mathrm{n}$ de b squeda utilizada, para la industria del deporte se hallaron 93.438 familias de patentes, y un total de 146.932 aplicaciones en los Itimos 20 a os. Del total de familias de patentes, 68.056 (73\%) se encuentran vigentes. El $67.59 \%$ corresponden a patentes de invenci n, el $8.31 \%$ a patentes de dise o industrial, el $19.69 \%$ a modelos de utilidad, y el $4.4 \%$ a patentes de proceso.

Las primeras diez jurisdicciones donde $\mathrm{m}$ s se protege la innovaci $\mathrm{n}$ en la industria del deporte son: China, con 40.230 
familias de patentes licenciadas; Estados Unidos, con 14.775; Alemania, con 11.270; Jap n, con 7.996; la Organizaci n Mundial de Patentes, con 6.287; Corea del Sur, con 5.738; la Oficina Europea de Patentes, con 5.223; Francia, con 4.844; Rusia, con 3.626; y Taiw n, con 3.166. A nivel latinoamericano, el pa s $\mathrm{m} \mathrm{s}$ innovador es Brasil, con 1.737. Colombia cuenta con 17 patentes vigentes registradas en su oficina local (ver figura 1).

La industria deportiva actual se encuentra concentrada en Asia, como el continente $\mathrm{m} \mathrm{s}$ innovador del mundo, con 57.130 familias de patentes licenciadas, que corresponden al $45 \%$ del total del mercado, a nivel mundial. China concentra el 32\% de las patentes, triplicando a Estados Unidos (12\%), Alemania (9\%) y Europa (5\%), en $n$ mero de familias de patentes. Se resalta que, a nivel Latinoamericano, solamente Brasil (1\%) se encuentra dentro de los 25 pa ses $\mathrm{m} \mathrm{s}$ innovadores en la industria deportiva internacional, seg n lo cual, la capacidad de innovaci n de la industria deportiva brasilera supera casi por 100 veces a la colombiana.

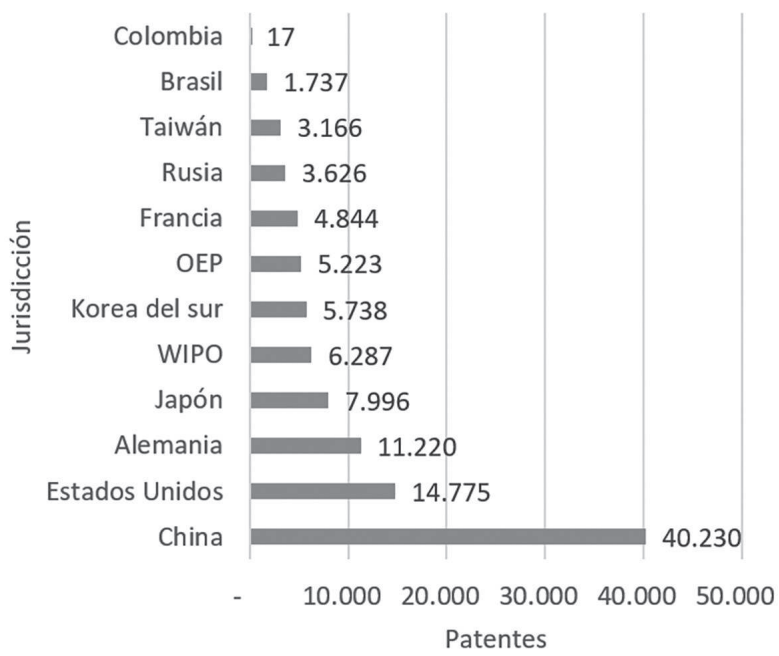

Figura 1. Número de patentes otorgadas de la industria deportiva por jurisdicción. Fuente: Patbase Analytics 2017 
La innovaci $n$ de la industria deportiva internacional ha venido aumentando de manera sostenida durante los Itimos 20 a os (ver figura 2). De 2007 a 2017 se ha duplicado el n mero de patentes otorgadas. Los incrementos $\mathrm{m}$ s notables en el registro de patentes coinciden con los a os previos y durante la realizaci n de los juegos ol mpicos y los mundiales de futbol. En Colombia, la primera patente se public en 1997, entre 2001 y 2010 se registraron 12 patentes, en 2016 una patente, y durante 2017 se publicaron 4 patentes. Los a os m s innovadores para la industria deportiva colombiana fueron 2007 (3 patentes) y 2017 (4 patentes). Se destaca que las patentes pertenecen a inventores y concesionarios locales, en su mayor a.

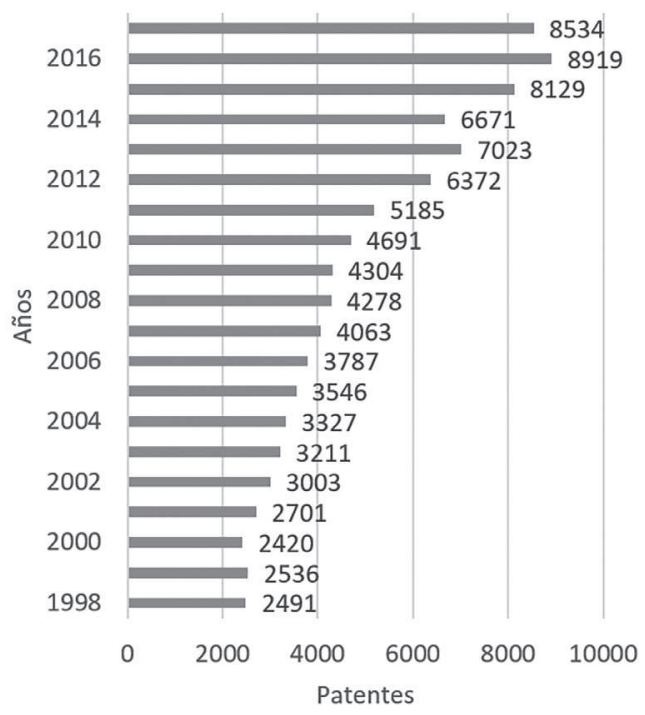

Figura 2. Número de patentes anuales otorgadas en la industria deportiva (1998-2017)

Fuente: Patbase Analytics 2017

La clasificaci n internacional de patentes (IPC), constituye un sistema jer rquico de s mbolos que no depende de idioma 
alguno para la clasificaci $\mathrm{n}$ de patentes y los modelos de utilidad que identifican los distintos sectores tecnol gicos. Esta clasificaci $n$ permite la consulta del estado de la $t$ cnica de manera actualizada cada a o (WIPO, 2016).

Las patentes otorgadas para la industria deportiva, se concentran en la familia A63B (23.875 patentes), que corresponde a la categor a de necesidades humanas (A); deportes-juegos-esparcimiento (63); aparatos para el entrenamiento f sico, gimn sticos, nataci n, escalada, esgrima, juegos de pelota y equipo de entrenamiento (B). En esta familia, las innovaciones se realizan, en su mayor a, sobre aparatos de entrenamiento para deportes especiales (A63B-69) (3.063 patentes), por ejemplo el paracaidismo; enseguida se encuentran dispositivos que permiten llevar el registro de scores para juegos o jugadores (A63B-71) (1.813 patentes); aparatos dise ados para ejercitar el sistema cardiovascular, la agilidad y la coordinaci $n$ (A63B-22) (1.000 patentes); y aparatos para entrenar la parte inferior del cuerpo (A63B-23) (938 patentes).

Las innovaciones para el calzado con prop sitos deportivos (A43B-05), cuenta con 2.688 patentes; partes estructurales del calzado (A43B-13), con 1.000 patentes; y botas para ski (A43B-5), con 688 patentes. En la familia A63C (4.188 patentes) se encuentran los dispositivos para skate, ski, roller skates, dise o o disposici $n$ de campos de juego. Se destacan las innovaciones en skateboards (A63C-17), con 1.000 patentes; el dise o de campos de juego (A63C-19), con 1.000 patentes; y los skis o snowboards (A63C-5), con 813 patentes. En la familia A41D (3.155 patentes) se encuentran todas las prendas y accesorios para protecci $\mathrm{n}$ de contacto f sico. En la familia B63B (2.313 patentes) se encuentran los equipamientos para navegaci n. Finalmente, en la familia G06F (1.875 patentes), se encuentran los dispositivos para el procesamiento de datos adaptados para cumplir funciones espec ficas, como estructuraci $\mathrm{n}$ de bases de datos y mediciones. En la tabla 2 se resumen las familias de patentes con mayor relevancia en la industria deportiva internacional. 
Tabla 2. Familias de patentes con mayor relevancia en la industria deportiva internacional (noviembre de 2017).

\begin{tabular}{|c|c|c|}
\hline IPC & Familia de patentes & Patentes \\
\hline A63B-69 & $\begin{array}{l}\text { Aparatos de entrenamiento para deportes } \\
\text { especiales }\end{array}$ & 3.063 \\
\hline A43B-05 & Calzado con propósitos deportivos & 2.688 \\
\hline B63B-35/73 & $\begin{array}{l}\text { Estructuras flotantes para deportes de } \\
\text { esparcimiento }\end{array}$ & 1.563 \\
\hline A43C-17 & Skateboards & 1.000 \\
\hline A41D-13 & $\begin{array}{l}\text { Prendas deportivas profesionales para } \\
\text { protección contra golpes o puños }\end{array}$ & 750 \\
\hline G06F-19 & $\begin{array}{l}\text { Computación digital o métodos de } \\
\text { procesamiento de datos }\end{array}$ & 563 \\
\hline
\end{tabular}

Fuente: Patbase Analytics 2017.

En lo relacionado con los cesionarios due os de los derechos de las patentes, se destaca la universidad de Yanshan, ubicada en Qinhuangdao, China, y cuenta con 358 familias de patentes en ingenier a de materiales para la industria deportiva. El cesionario Salomon, de Francia, cuenta con 358 familias de patentes, y se especializa en fabricar productos y accesorios para carrera, monta ismo, senderismo, esqu y snowboard. La empresa japonesa de indumentaria e implementos deportivos Mizuno, tiene $118 \mathrm{fa}-$ milias de patentes. Nike, con 103 familias de patentes, se mantiene como una de las compa as $\mathrm{m}$ s innovadoras del mundo en lo relacionado con calzado e indumentaria deportiva. La universidad de Shandong, en China, posee 102 familias de patentes. Finalmente, Adidas cuenta con 86 familias de patentes registradas, en su mayor a en la oficina alemana y europea.

En Colombia existen 17 familias de patentes registradas. El potencial competitivo se observa en aparatos para el entrenamiento $f$ sico, partes para calzado deportivo, partes para bicicleta, accesorios y partes para deportes motorizados y botes, 
resultados que se alinean con los segmentos de la industria deportiva internacional. Aunque no registran patentes, tambi $n$ se identific potencial competitivo en implementos y accesorios para roller sports y en software para e-sports. En la tabla 3 se describen las familias de patentes (11) que predominan en la industria deportiva colombiana y, por lo tanto, cuentan con potencial de competitividad en los mercados internacionales.

Tabla 3. Familias de patentes en Colombia.

\begin{tabular}{llc}
\hline Familia de patentes & Tipo de patente & Numero \\
\hline A43 B & $\begin{array}{l}\text { Suelas y plantillas para calzado } \\
\text { deportivo } \\
\text { A63 B }\end{array}$ & 4 \\
A61 H & $\begin{array}{l}\text { Pelotas y flotadores para natación } \\
\text { Boccia }\end{array}$ & 1 \\
A63 F & $\begin{array}{l}\text { Cancha de mini tejo } \\
\text { B62K }\end{array}$ & 1 \\
B63B & Partes para bicicleta & 1 \\
B62D & Accesorios y partes para botes & 1 \\
\hline
\end{tabular}

Fuente: Patbase Analytics 2017

\section{CONCLUSIONES}

China es el epicentro de la industria deportiva, y sus indicadores de innovaci $\mathrm{n}$ son significativamente superiores a los de Estados Unidos y Europa. Los inventores individuales chinos son quienes poseen el mayor $n$ mero de patentes, lo que denota que la mayor a se encuentran en estadio de invenci $n$ y carecen de alg $n$ valor comercial. Por otro lado, las universidades chinas son las

5 Deporte aut ctono de la regi $\mathrm{n}$ andina colombiana. 
principales desarrolladoras de procesos de innovaci $\mathrm{n}$ tecnol gica deportiva, aunque ello no implica que los niveles de innovaci $\mathrm{n}$ de la industria deportiva China sean superiores a las del resto del mundo. Las empresas internacionales con mejores indicadores de innovaci n como Salomon, Mizuno, Nike y Adidas, registran la mayor a de sus invenciones en sus lugares de origen.

Al parecer, son los mega eventos deportivos los grandes movilizadores de la innovaci $\mathrm{n}$ en la industria deportiva contempor nea, lo que se evidencia en el incremento significativo del registro de patentes en los a os previos y durante su realizaci n. El registro de patentes tuvo un incremento del 100\% en los Itimos diez a os en la industria deportiva internacional, pasando de 4.063 a 8.534 registros durante el periodo 20072017. El deporte global es un mercado intensivo en tecnolog a, que dispone del espect culo deportivo como escenario para la validaci $\mathrm{n}$ y difusi $\mathrm{n}$ de las innovaciones.

La industria deportiva internacional innova intensivamente en aparatos para el entrenamiento deportivo y el calzado deportivo, coincidiendo con las capacidades actuales de innovaci $n$ de la industria deportiva colombiana. Esto se presenta como un factor $\mathrm{cr}$ tico de xito para tener en cuenta en la formulaci $\mathrm{n}$ de pol ticas focalizadas en innovaci n deportiva, el dise o de programas de desarrollo cient fico y tecnol gico, y la realizaci $n$ de convocatorias para financiar el desarrollo de la innovaci $n$ en la industria deportiva basadas en las capacidades locales y en los segmentos priorizados del mercado deportivo global.

\section{Investigación futura}

Las principales orientaciones para la investigaci $\mathrm{n}$ futura relacionadas con la propiedad intelectual en el contexto del deporte, se relacionan con las tendencias, limitaciones y problem ticas que subyacen en la concepci $\mathrm{n}$ del deporte como un bien $\mathrm{p}$ blico, y hasta qu nivel se puede considerar la protecci $n$ de la innovaci n como una limitante para el desarrollo deportivo. 
Se debe profundizar en el estudio del balance que debe existir entre la propiedad intelectual y el inter s p blico como uno de los principios que oriente los procesos de desarrollo deportivo. El principal argumento es que algunas marcas relacionadas con las organizaciones deportivas, se pueden considerar como un bien $\mathrm{p}$ blico, debido a que sus or genes se encuentran fuertemente arraigados en grupos sociales que han consolidado su posicionamiento, gracias a la tradici n, y c mo la protecci $n$ de estas limita el acceso de la poblaci $n$ en general al deporte.

Por otro lado, resulta necesario estudiar el desarrollo de prototipos dentro de las comunidades que desarrollan las pr cticas deportivas, y c mo este proceso permite identificar potenciales innovaciones. En este sentido, es necesario estudiar c mo las organizaciones deportivas pueden convertir en activos el registro de marcas, el registro de patentes, el registro de software, los dise os industriales y los planos de instalaciones deportivas.

\section{REFERENCIAS}

1. ACCI Generalitat de Catalunya (2015). La ind stria de l'Esport a Catalunya. Update 2015. Espa a: Generalitat de Catalunya.

2. CAN Comunidad Andina de Naciones (1993). Decisión 351-R gimen com $n$ sobre derechos de autor y derechos conexos. Lima: Comunidad Andina de Naciones.

3. Cantrell, R. (1999). The six angles of competition. Competitive Intelligence Review, 10(3), 51-57.

4. Ceballos, R. (2014). Los tratados de libre comercio y los derechos de propiedad intelectual: una pol tica de integraci n comercial en Colombia. Revista del Instituto de Ciencias Jurídicas de Puebla, 8(33), 223-256.

5. Comerford, S. (2012). International intellectual property rights and the future of global e-sports. Brooklyn Journal of International Law, 37(2), 623-648.

6. de Beer, J. (2015). "Open" innovation policy frameworks: Intellectual property, competition, investment \& other market governance issues. Ottawa: Industry Canada.

7. de Beer, J. (2016). Evidence - based intellectual property policy making: An integrated review of methods and conclusions. Journal of World Intellectual Property, 19(5-6), 150-177. 
8. D az, M., Guzm n, M., \& Orea, U. (2007). Estudio patentom trico de un proyecto de investigaci n. Ciencias de la Informaci n, 38(1-2), 57-66.

9. Dutta, S., Lanvin, B., \& Wunch, S. (2016). THe Global Innovation Index 2016: Winning with Global Innovation. Geneve: Cornell University, INSEAD and WIPO.

10. Espinal, D. (2018). Prospectiva del desarrollo deportivo: visiones de futuro del sistema deportivo colombiano (Tesis doctoral). Santiago de Cali: Universidad del Valle.

11. Estrada, S. (2015). Implicaciones económicas de la tecnociencia en el deporte. En: L. Rodr guez, I. valos, \& I. Vega (eds.), Tecnociencia, deporte y sociedad: ¿victorias de laboratorio? Vol. III (pp.60-94). Bogotá: Corporaci n Colombia Digital.

12. EC European Comission (2013). Europa Sport satellite acount. Brussels: European Comission.

13. Harari, Y. (2016). Homo deus: breve historia del ma ana. Madrid: Debate

14. Herstein, R., \& Berger, R. (2013). City rebranding: Sports events as image builder. Journal of Business Strategy, 34(3), 21-39.

15. Kalamadi, S. (2012). Intellectual property and the business of sport management. Journal of Intellectual Property Rights, 17, 437-442.

16. Kief, S., Kramer, S., \& Kunstadt, R. (2009). It's your turn, but it's my move: Intellectual property protection for sports moves. Santa Clara High Technology Law Journal, 25, 765-785.

17. Li, M., Macintosh, E., \& Bravo, G. (2012). International sport management. Champaign, IL: Human Kinetics.

18. Marketline (2014). Sports Equipment: Global Industry Guide. London: Marketline.

19. Mateska, E. (2016). Activities of the customs administration for protecting the intellectual property rights. Balkan and Near Eastern Journal of Social Sciences, 2(4), 128-133.

20. Milano, M., \& Chelladurai, P. (2011). Gross domestic sport product: The size of the sport industry in the United States. Journal of Sport Management, 25, 24-35.

21. Ming, Y., \& Si Hu, K. (2013). Empirical research on the sports equipment of international application for patent competitive intelligence between China and United States. Journal of Beijing Sport University, 4, 11-16.

22. Plunkett, J. (2017). Global sports industry revenues 2015-2016. In: J. Plunkett, Plunkett's sports industry almanac 2018: Sports \& recreation industry market research, statistics, trends \& leading companies (p.38). USA: Plunkett Research.

23. PWC Price Waterhouse Coopers (2015). Changing the game. Outlook for the global sports market to 2015. London: PWC. Available from: 
https://www.pwc.com/gx/en/hospitality-leisure/pdf/changing-the-game-outlook-for-the-global-sports-market-to-2015.pdf

24. Ratten, V. (2011). Sport-based entrepreneurship: Towards a new theory of entrepreneurship and sport management. International Entrepreneurship and Management Journal, 7(1), 57-69.

25. Ratten, V., \& Ferreira, J. (2016). Sport entrepreuneurship and innovation. London: Routledge.

26. Schaefer, J. (2012). Sport and intellectual property. Geneve, Switzerland: WIPO. Available from: http://www.wipo.int/ip-sport/en/

27. Schwab, K., \& Sala-i-Martin, X. (2016). The global competitiveness report 2016-2017. Geneve, Switzerland: World Economic Forum.

28. Skinner, J., Edwards, A., \& Corbett, B. (2015). Research methods for sport management. New York: Routledge.

29. Taylor, W. (2016). Global intellectual property index: 5th report. London: Taylor Wessing. Available from: https://united-kingdom.taylorwessing. com/documents/get/576/gipi5-report.pdf/show_on_screen

30. Tjønndal, A. (2016). Sport, innovation and strategic management: A systematic literature review. Brazilian Business Review, 13 (SpEd), 38-56.

31. Universidad del Valle (2003). Acuerdo 023 de 2003 - Estatuto de propiedad intelectual. Cali: Universidad del Valle.

32. MCVET Methodological Centre for Vocational Education and Training (2008). Study of sports sector. Research report on skill needs. Vilnius, Lithuania: MCVET. Available from: http://www.kpmpc.It/Skelbimai/ SEK_EN/EN-Sporto\%20sekt.\%2008.07.30.pdf

33. Winand, M., \& Hoeber, L. (2016). Innovation capability of non-profit sport organizations. In: V. Ratten \& J. Ferreira, Sport entrepreneurship and innovation (pp.40-63). London: Routledge.

34. WIPO World Intellectual Property Organization (2016). What is inteIlectual property? Geneve, Switzerland: WIPO.

35. Zheng, L. (2011). The research of identification and determination for sports industry cluster. In: International Conference on Management and Service Science, MASS 2011. 